\title{
Exploring the Possibility of Deformation Twinning in Pure Aluminum
}

\author{
Tuo Cai ${ }^{1} \cdot$ Zhen-Jun Zhang $^{1} \cdot$ Jin-Bo Yang ${ }^{1} \cdot$ Zhe-Feng Zhang $^{1}$
}

Received: 9 March 2015/Revised: 10 April 2015/Published online: 31 May 2016

(c) The Chinese Society for Metals and Springer-Verlag Berlin Heidelberg 2016

\begin{abstract}
Can aluminum generate deformation twins? Contradictories exist among the experimental results, theoretical predictions, and molecular dynamics simulations. Our first-principles calculations based on the full-potential linearly augmented plane wave method show that the bonding electron density of aluminum gathers at the tetrahedral interstices, and this specific electronic structure is in agreement with the experimental data from quantitative convergent-beam electron diffraction. In terms of this characteristic, the matching between the cohesive energy of local deformation twins with different thickness and global matrix can shed light on deformation twins in aluminum. This study may give a lane to insight into deformation twins in different metals.
\end{abstract}

KEY WORDS: Metals and alloys; Deformation twinning; First-principles; Density functional theory

\section{Introduction}

Deformation twinning (DT) is a basic deformation mechanism in many materials such as pure metals, steels, shapememory alloys, rocks, ferroelectric, and ferromagnetic materials [1-5]. In general, a consensus is that the preference for DT in face-centered cubic (fcc) metals depends largely on their stacking fault energies (SFEs). For instance, coarse-grained $\mathrm{Cu}, \mathrm{Al}$, and $\mathrm{Ni}$ with medium-tohigh SFE prefer to deform via dislocation slip at ambient temperature and low strain rate; while $\mathrm{Ag}$ and $\mathrm{Au}$ with low SFE can deform by DT $[6,7]$. In addition, the addition of $\mathrm{Al}$ into $\mathrm{Cu}$ will make it easier to generate deformation twins in $\mathrm{Cu}-\mathrm{Al}$ alloys owing to the decrease of SFE $[8,9]$.

Available online at http://link.springer.com/journal/40195

Tuo Cai

zhfzhang@imr.ac.cn

1 Shenyang National Laboratory for Materials Science, Institute of Metal Research, Chinese Academy of Sciences, Shenyang 110016, China
Generally, SFE is regarded as a critical parameter that determines whether DT happens or not. In many fcc metals and alloys, there is a reasonable tendency to form deformation twins with the decrease of SFE. Among the previous studies, DT was observed in Ir, while DT was rarely reported in bulk Al samples, although its SFE is lower than Ir [10]. In addition, three criteria present the similar result that $\mathrm{Al}$ can hardly deform via DT. The first criterion was proposed a decade ago by Bernstein and Tadmor [11] to predict twinning tendency based on the heterogeneous dislocation nucleation from a crack tip and the generalized SFE curve. The second one was proposed by Asaro and Suresh [12] in terms of the inhomogeneous dislocation nucleation from a grain boundary and the generalized SFE curve. The third one was proposed by Cai et al. [13] to predict the twinning tendency in the framework of the generalized SFE curve. However, molecular dynamics (MD) simulations [14-16] demonstrate that DT can still occur in Al with a sufficiently small grain size.

In addition to the theoretical predictions and MD simulations, various experiments have been performed to investigate the DT mechanism in single-crystalline, coarse- 
grained, and nanocrystalline Al. Firstly, almost no deformation twins have been experimentally observed in single or coarse-grained pure $\mathrm{Al}$ [10], even under shock loading at very low temperatures [17]. Secondly, the occurrence of Shockley partial dislocations from a crack tip of $\mathrm{Al}$ has been regarded as an evidence of DT; however, more detailed evidences supporting this result have not been observed again. Thirdly, deformation twins have been observed in nanocrystalline $\mathrm{Al}$ after severe plastic deformation [18-22]. The last one is that microtwins have been observed in single-crystalline $\mathrm{Al}$ via equal-channel angular pressing (ECAP) with the perfect crystallographic orientation design [23]. In contrast to these observations, twinning and detwinning have been captured for polycrystalline Al with grain sizes from several micrometers to $200 \mu \mathrm{m}$ in tensile experiment [24].

According to the results above, there are some mismatches among different experimental results, MD simulations, and different theoretical predictions for the generation of deformation twins in Al. Firstly, the three criteria present the similar prediction results that DT can hardly occur in $\mathrm{Al}$ [11-13], while MD simulations illustrate the presence of deformation twins in $\mathrm{Al}$ with a very small grain size [14-16]. Secondly, DT can occur in singlecrystalline and nanocrystalline Al [18-22], whereas twinning and detwinning have been captured in coarse-grained $\mathrm{Al}$ [24]. It is obvious that those theoretical predictions and MD simulations are not in good agreement with the experimental results. These analyses above give rise to an interesting question whether $\mathrm{Al}$ can deform via DT or not. Is there a channel to understand and explicate the contradictions between the theoretical prediction and experimental results?

In view of those contradictions above, a computational model is proposed in the present study based on a fundamental hypothesis that a successive deformation twin is generated in $\mathrm{Al}$ matrix. And then the cohesive energy of this deformation twin with different thickness is calculated by first-principles method, which is based on the full-potential linearly augmented plane wave (FPLAPW) method incorporating local orbital in the framework of density functional theory (DFT) as packaged in WIEN2k code [25]. In succession, the difference between the cohesive energy of fcc $\mathrm{Al}$ and a successive deformation twin with different thickness in the range from 0.5 to $7 \mathrm{~nm}$ is responsible for whether $\mathrm{Al}$ can deform via DT or not. This method has been used to solve other problems previously $[26,27]$. Finally, the particular electronic structure of fcc $\mathrm{Al}$ is that the bonding electron density locates at the tetrahedron interstices, which has been confirmed by the quantitative convergent-beam electron diffraction (QCBED) experiment [28].

\section{Computation Methods}

The present computational results are calculated by means of FPLAPW method incorporating local orbital within DFT which is packaged in WIEN2k code [25]. The generalized gradient approximation (GGA) of Perdew et al. [29] is used for exchange-correlation energy functional. And the muffin-tin radius of $\mathrm{Al}$ atom is $2.0 \mathrm{Bohr}$. The size of the basis set is given by the product $R_{\mathrm{mt}} \times K_{\max }=8.0$, where $K_{\max }$ is the largest reciprocal space wave vector in the basis set and $R_{\mathrm{mt}}$ is the smallest muffin-tin radius of atom. The separation energy between inner electrons and valence electrons is $-81.6 \mathrm{eV}$ in the calculation of exchange-correlation energy. The energy accuracy is thus less than $0.00001 \mathrm{eV} .3000 \mathrm{k}$ points can converge our total energy in the first Brillouin zone for all computations. The calculated bulk properties of $a=4.038 \AA, B=72.6 \mathrm{GPa}$, and $E_{\text {coh }}=3.517 \mathrm{eV}$ (lattice constant, bulk modulus, and cohesive energy of $\mathrm{Al}$, respectively) are consistent with the experimental results of $a=4.05 \AA$ [30], $B=72.2 \mathrm{GPa}$ [31], and $E_{\mathrm{coh}}=3.39 \mathrm{eV}$ [32].

\section{Results and Discussion}

The computational mode is illustrated in Fig. 1. The mode is built along $x, y$, and $z$ directions, where $x$ is equal to one $\frac{1}{2}\langle\overline{1} 10\rangle, y$ is equal to one $\frac{1}{6}\langle 11 \overline{2}\rangle$, and $z$ is equal to $2 h$ along the $\langle 111\rangle$ direction. This mode shows successive twins with the thickness $h$, and...ABCACBA... layer-to-layer

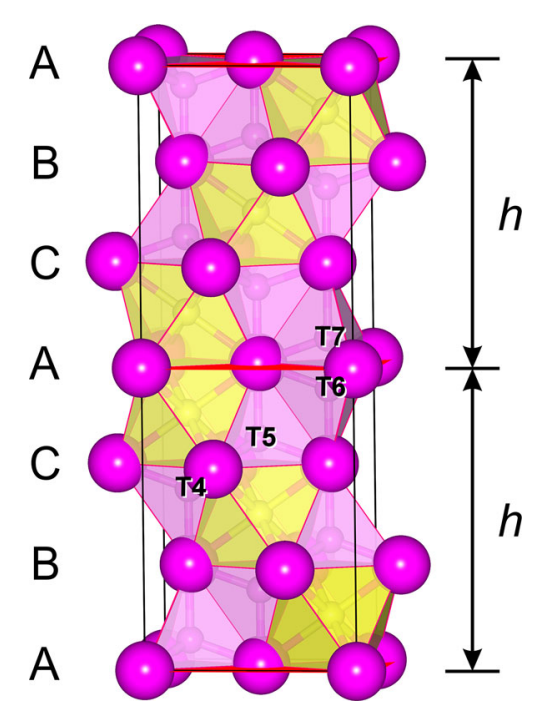

Fig. 1 Computational mode of twinned $\mathrm{Al}$ with thickness $h$. The structure is composed of layers A, B, C, A, C, B, A, filled by the tetrahedral interstices that are colored with the light magenta and the octahedral interstices that are colored with the bright yellow, in which T4, T5, T6 and T7 are the representative tetrahedral interstice sites 
structure, in which A represents a twin boundary (TB). The thickness $h$ is a successive variable, which can adjust the thickness of deformation twins. A series of successive deformation twins are constructed, the cohesive energy of these models with different thickness is calculated by the first-principles method, and the computational results are presented in Fig. 2. It is clear that the cohesive energy of deformation twins gradually increases as $h$ increases layer by layer. When $h$ increases to about $3 \mathrm{~nm}$, the cohesive energy converges at the about $3.5 \mathrm{eV}$, which is still apart from that of fcc $\mathrm{Al}$ [32]. Although the cohesive energy of the deformation twin converges at one equilibrium state when $h$ is greater than $3 \mathrm{~nm}$, it is not a stable structure in contrast to perfect fcc $\mathrm{Al}$, which is just a metastable state. In view of this, even if DT can occur in single-crystal Al and deformation twins can generate layer by layer, no matter how thick it grows up, the cohesive energy of the local deformation twins cannot arrive at that of the global fcc Al. Therefore, the difference between the cohesive energy of local deformation twins and global fcc Al results in that the equilibrium state for deformation twins disappears through inverse twinning, i.e., detwinning, as observed in the previous study [24], unless the samples are Al film [18] and nanocrystalline Al [19-21].

Why the cohesive energy of deformation twins cannot approach to the value of prefect fcc Al with increasing $h$ ? In view of the particular electronic structure of $\mathrm{Al}$, this question should be well resolved. The deformation electron density $\Delta \rho$ is defined as below,

$\Delta \rho=\rho_{\text {total }}-\rho_{\text {IAM }}$

where $\rho_{\text {total }}$ is the total electron density and $\rho_{\text {IAM }}$ is the density associated with free, unbonded atoms, otherwise known as the independent atom model (IAM) [33]. The iso-surface and contour of $\Delta \rho$ in fcc $\mathrm{Al}$ are illustrated in Fig. 3. It is obviously shown that the $\Delta \rho_{\text {tet }}$ (the deformation electron density at the centers of the tetrahedral interstices) is greater than the $\Delta \rho_{\text {oct }}$ (the deformation electron density at the centers of the octahedral interstices) and the positive $\Delta \rho$ nearly concentrates at the tetrahedral interstices, which is in good agreement with the experimental result by means of QCBED method [28].

Similarly, the deformation electron density $\Delta \rho$ of a deformation twin is illustrated in Fig. 4. It is found in Fig. 1 that the bridge between the tetrahedral interstices can merely rely on the atom (a point) or edge (line) between the nearest atoms and never depend on the lattice plane in the fcc $\mathrm{Al}$ [28]. However, this rule is broken when the DT occurs in fcc Al. The bridge between the tetrahedral interstices can also rely on lattice plane, such as a $\langle 111\rangle$ plane, because TBs are generated in fcc matrix. In general, the relative positions between T6 (a tetrahedral interstice) and T7 (another tetrahedral interstice) are similar to those between T4 (a tetrahedral interstice) and T5 (a tetrahedral interstice) which are linked via an edge; however, after DT occurs, both T6 and T7 are linked via a lattice plane (TB plane). These changes directly result in the decrease in the distance between T6 and T7 from $a / 2$ to $\sqrt{3} a / 6$ ( $a$ is the lattice constant of $\mathrm{fcc} \mathrm{Al})$. Therefore, the original repulsive force between T6 and T7 is expressed as follows:

$F_{0}=k \frac{\Delta \rho_{T 6} \times \Delta \rho_{T 7}}{(a / 2)^{2}}$,

and the resultant repulsive force between $\mathrm{T} 6$ and $\mathrm{T} 7$ is given as:
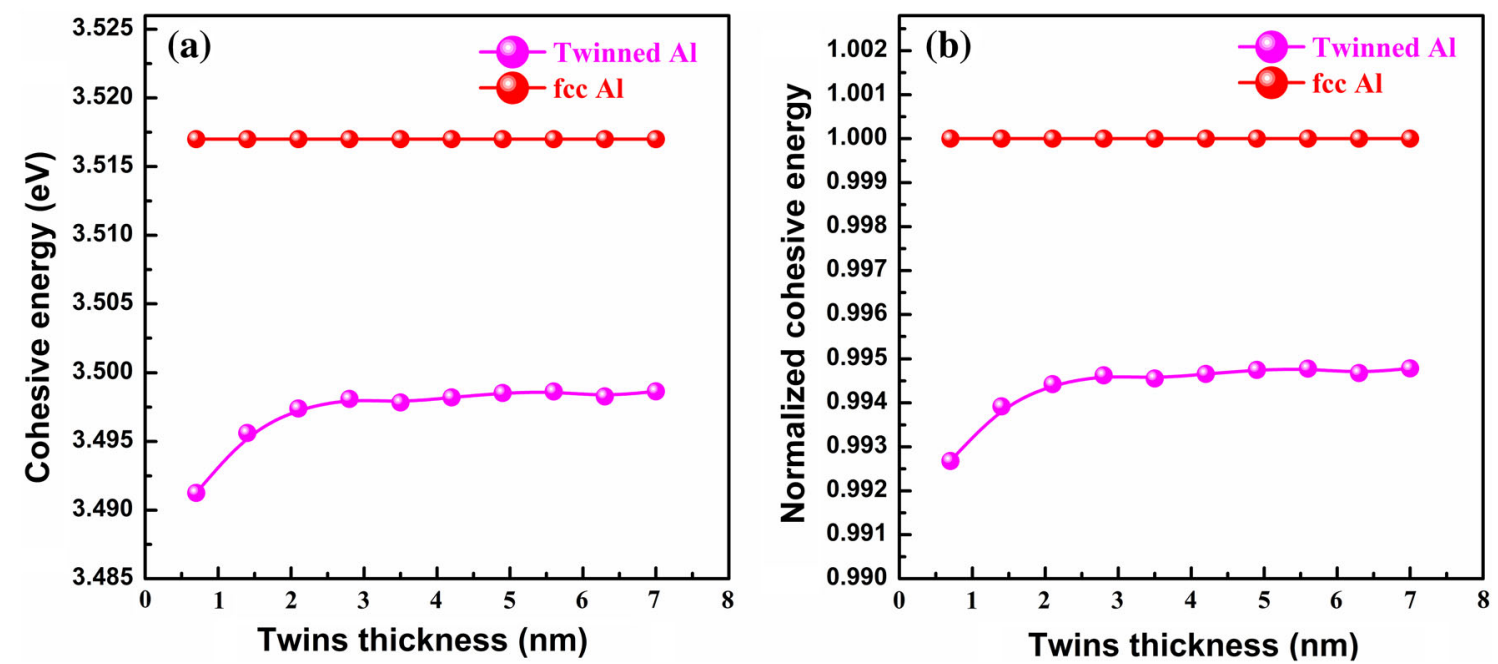

Fig. 2 Comparison between the cohesive energy of twinned $\mathrm{Al}$ with different thickness and a perfect fcc $\mathrm{Al}$ : a original cohesive energy and b normalized cohesive energy 


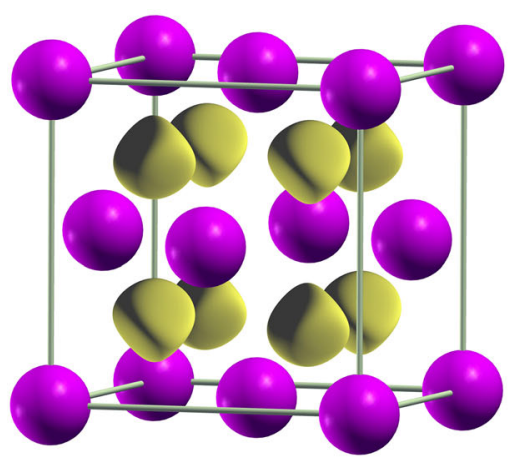

(a)

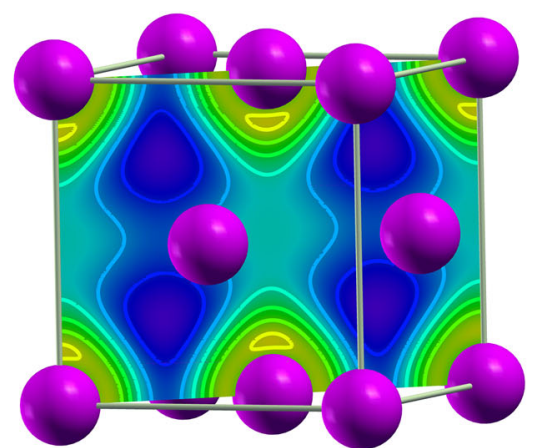

(b)

Fig. 3 a Iso-surface value $\Delta \rho$ with $0.0275 \mathrm{e} / \AA^{3}$ in the unit cell of $\mathrm{Al}$, located at the tetrahedral interstice. b Contour of $\Delta \rho$ in $\{110\}$ planesectioned planes including the tetrahedral and octahedral interstices. The contours are the six intervals from -0.075 to 0.055 e/ $\AA^{3}$

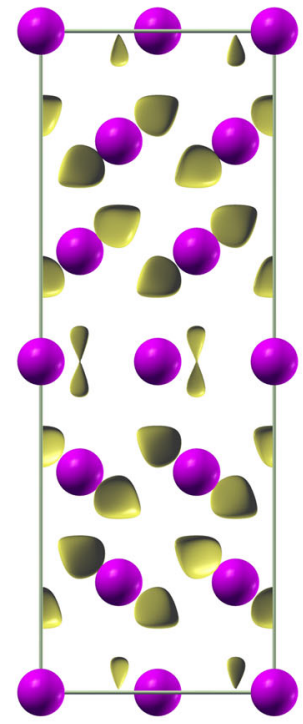

(a)

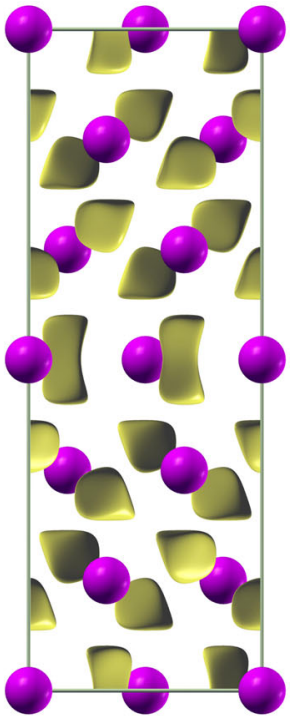

(b)

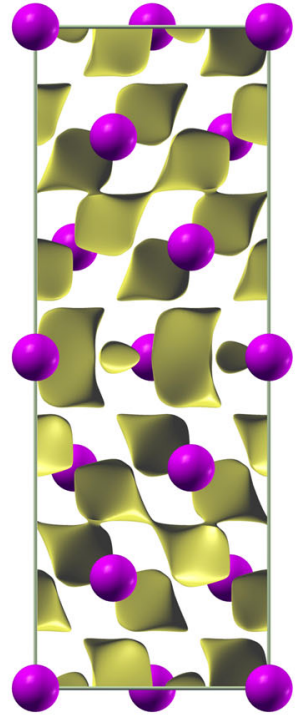

(c)

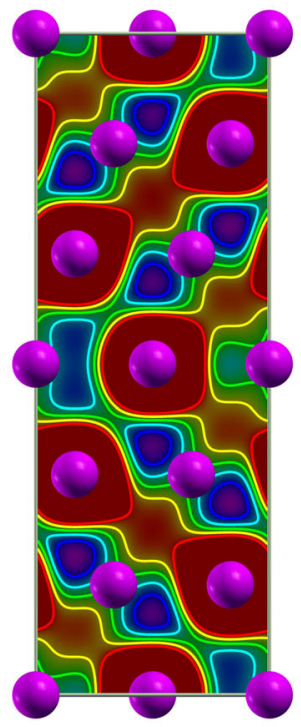

0.000

$\square 0.008$

0.016

$\square 0.024$

0.032

0.040

Fig. 4 Contour of iso-surfaces for $\mathbf{a} \Delta \rho=0.0284 \mathrm{e} / \AA^{3}, \mathbf{b} \Delta \rho=0.0231 \mathrm{e} / \AA^{3}$, and $\mathbf{c} \Delta \rho=0.0184 \mathrm{e} / \AA^{3}$, respectively. d Contour of $\Delta \rho$ in planesectioned plane including the tetrahedral and octahedral interstices. The contours are the six intervals from 0 to $0.04 \mathrm{e} / \AA^{3}$

$F_{1}=k \frac{\Delta \rho_{T 6} \times \Delta \rho_{T 7}}{(\sqrt{3} a / 6)^{2}}$,

where $k$ is the Coulomb constant. Because $F_{0}<F_{1}$, the cohesive energy of deformation twins is lower than that of fcc Al.

In Fig. 4a, there are two small bonding electron densities in the adjacent of TB plane. As the value of iso-surface gradually increases, these bonding electron densities fuse into one bonding electron density $\Delta \rho_{1}$ from Fig. $4 \mathrm{~b}$, and then $\Delta \rho_{1}$ gets bigger and another bonding electron density $\Delta \rho_{2}$ appears in the TB plane and near $\Delta \rho_{1}$ from Fig. $4 \mathrm{c}$. $\Delta \rho_{1}$ is defined as double-core-pseudo-charge, and $\Delta \rho_{2}$ is also defined as extra-pseudo-charge. The repulsive force between double-core-pseudo-charge $\Delta \rho_{1}$ and extra-pseudo- charge $\Delta \rho_{2}$ minifies the cohesive energy of deformation twins because these repulsive forces and TBs are symbiotic in fcc $\mathrm{Al}$ matrix.

Although the cohesive energy of local deformation twins is far smaller than that of the global fcc Al matrix, the cohesive energy of global matrix decreases when the grain size is smaller (the global matrix changes from singlecrystalline to coarse-grained and nanocrystalline $\mathrm{Al}$ ), because the volume fraction of atoms in the grain boundaries (GBs) gets larger and larger, and the energy of atoms in GB is higher than that of atoms in bulk sample. Why could twinning and detwinning be captured in the coarsegrained crystalline $\mathrm{Al}$ ? Because the cohesive energy of the coarse-grained crystalline $\mathrm{Al}$ with grain sizes ranging from several micrometers to $200 \mu \mathrm{m}$ is still smaller than that of 
the deformation twins, the stability of local deformation twins can match that of global Al matrix [24]. As a result, it is not difficult to understand why deformation twins were observed in the nanocrystalline $\mathrm{Al}$ film with thickness of 200-400 nm [19-21], and a grain size of 10-35 nm [18]. The latest experimental result [34] presents that deformation twins are captured in single $\mathrm{Al}$; however, the figure shows that the deformation twins are also embedded in polycrystalline $\mathrm{Al}$, and the grain size just is large.

\section{Conclusions}

The matching of cohesive energy between the local deformation twins and the global matrix in $\mathrm{Al}$ should be responsible for the contradictories among the experimental results, theoretical predictions, and MD simulations. Firstly, when the cohesive energy of deformation twins is far smaller than that of the single- and coarse-grained crystalline $\mathrm{Al}$, the deformation twins in the matrix should behave in detwinning synchronously. Secondly, when the cohesive energy of local deformation twins is close to that of global nanocrystalline $\mathrm{Al}$, deformation twins can form in nanocrystalline Al film. In nature, the particular electronic structure of $\mathrm{Al}$ that bonding electron density gathers at the tetrahedral interstices underlays the inconsistency between the experimental results and theoretical predictions for the formation of deformation twins in Al.

Acknowledgments We acknowledge the support from the National Natural Science Foundation of China (NSFC) under grant Nos. 51101162, 51201165, and 51331007. We thank A. Shah, Y.Z. Tian, H.L. Wang, and B. Wang for language revision and improvements. J.B. Yang would like to acknowledge the financial support of "Hundred of Talents Project" by the Chinese Academy of Sciences.

\section{References}

[1] J.W. Christian, S. Mahajan, Prog. Mater Sci. 39, 1 (1995)

[2] D.H. Ping, Acta Metall. Sin. (Engl. Lett.) 27, 1 (2014)

[3] X.L. Wang, W.B. Dai, R. Wang, X.Z. Tian, X. Zhao, J.M. Li, Acta Metall. Sin. (Engl. Lett.) 27, 267 (2014)

[4] M.H. Li, Y.Q. Yang, M. Han, W. Zhang, B. Huang, X. Luo, J.G. $\mathrm{Ru}$, Acta Metall. Sin. (Engl. Lett.) 27, 667 (2014)

[5] S.F. Chen, L. Zheng, S.H. Zhang, H.W. Song, M. Cheng, Acta Metall. Sin. (Engl. Lett.) 28, 1426 (2015)
[6] F.J. Humphreys, M. Hatherly, Recrystallization and Related Annealing Phenomena, 2nd edn. (Pergamon, New York, 1995)

[7] S.H. Zhang, S.F. Chen, Y. Ma, H.W. Song, M. Cheng, Acta Metall. Sin. (Engl. Lett.) 28, 1452 (2015)

[8] S. Qu, X.H. An, H.J. Yang, C.X. Huang, G. Yang, Q.S. Zang, Z.G. Wang, S.D. Wu, Z.F. Zhang, Acta Mater. 57, 1586 (2009)

[9] X.H. An, Q.Y. Lin, S.D. Wu, Z.F. Zhang, R.B. Figueiredo, N. Gao, T.G. Langdon, Scr. Mater. 64, 954 (2011)

[10] J.P. Hirth, H.C. Rogers, Deformation Twinning (Gordon and Breach, New York, 1964)

[11] N. Bernstein, E.B. Tadmor, Phys. Rev. B 69, 094116 (2004)

[12] R.J. Asaro, S. Suresh, Acta Mater. 53, 3369 (2005)

[13] T. Cai, Z.J. Zhang, P. Zhang, J.B. Yang, Z.F. Zhang, J. Appl. Phys. 116, 163512 (2014)

[14] V. Yamakov, D. Wolf, S.R. Phillpot, A.K. Mukherjee, H. Gleiter, Nat. Mater. 1, 45 (2002)

[15] A. Froseth, H. Van Swygenhoven, P.M. Derlet, Acta Mater. 52, 2259 (2004)

[16] K.W. Jacobsen, J. Schiøtz, Nat. Mater. 1, 15 (2002)

[17] G.T. Gray, III. Acta Metall. 36, 1745 (1988)

[18] M.W. Chen, E. Ma, K.J. Hemker, H.W. Sheng, Y.M. Wang, X.M. Cheng, Science 300, 1275 (2003)

[19] X.Z. Liao, F. Zhou, E.J. Lavernia, S.G. Srinivasan, M.I. Baskes, D.W. He, Y.T. Zhu, Appl. Phys. Lett. 83, 632 (2003)

[20] X.Z. Liao, F. Zhou, E.J. Lavernia, D.W. He, Y.H. Zhu, Appl. Phys. Lett. 83, 5062 (2003)

[21] Y.T. Zhu, X.Z. Liao, S.G. Srinivasan, Y.H. Zhao, M.I. Baskes, F. Zhou, E.J. Lavernia, Appl. Phys. Lett. 85, 5049 (2004)

[22] X.L. Wu, X.Z. Liao, S.G. Srinivasan, F. Zhou, E.J. Lavernia, R.Z. Valiev, Y.T. Zhu, Phys. Rev. Lett. 100, 095701 (2008)

[23] W.Z. Han, G.M. Cheng, S.X. Li, S.D. Wu, Z.F. Zhang, Phys. Rev. Lett. 101, 115505 (2008)

[24] B.Q. Li, M.L. Sui, B. Li, E. Ma, S.X. Mao, Phys. Rev. Lett. 102, 205504 (2009)

[25] P. Blaha, K. Schwarz, G.K.H. Madsen, D. Kvasnicka, J. Luitz, Computer Code WIEN2 K, An Augmented Plane Wave Plus Local Orbital Program for Calculating Crystal Properties (Vienna University of Technology, Vienna, 2014)

[26] R. Wu, A.J. Freeman, G.B. Olson, Science 265, 376 (1994)

[27] M. Yamaguchi, M. Shiga, H. Kaburaki, Science 307, 393 (2005)

[28] P.N.H. Nakashima, A.E. Smith, J. Etheridge, B.C. Muddle, Science 331, 1583 (2011)

[29] J.P. Perdew, K. Burke, M. Ernzerhof, Phys. Rev. Lett. 77, 3865 (1996)

[30] H.E. Swanson, Standard X-ray Diffraction Power Patterns, (U.S. GPO, Washington, DC, 1953)

[31] G. Simmons, H. Wang, Single Crystal Elastic Constants and Calculated Aggregate Properties: A Handbook, (MIT Press, Cambridge, MA, 1971)

[32] C. Kittel, Introduction to Solid State Physics (Wiley, New York, 1996)

[33] P.A. Doyle, P.S. Turner, Acta Crystallogr. A 24, 390 (1968)

[34] F. Zhao, L. Wang, D. Fan, B.X. Bie, X.M. Zhou, T. Suo, Y.L. Li, M.W. Chen, C.L. Liu, M.L. Qi, M.H. Zhu, S.N. Luo, Phys. Rev. Lett. 116, 075501 (2016) 